\section{$121 \quad$ CHANGES IN ENERGY LOSS AND PRESSURE RECOVERY AFTER BIOPROSTHESIS REPLACEMENT FOR AORTIC STENOSIS}

X Y Jin, ${ }^{1}$ L Yuan, ${ }^{1} \mathrm{~J} T \mathrm{Hu},{ }^{1} \mathrm{C}$ Ratnatunga ${ }^{2}{ }^{1} \mathrm{NDCLS}$, RDM, Oxford University \& Cardiac Surgery, John Radcliffe Hospital; ${ }^{2}$ Cardiac Surgery, John Radcliffe Hospital

doi:10.1136/heartjnl-2013-304019.121

Objectives Energy loss was shown to provide a more accurate assessment than pressure gradient or effective orifice area for aortic stenosis or prosthesis, as the former takes the pressure recovery into account while the later only measures the vena contracta orifice area. The present study aimed to elucidate the medium term changes in aortic prosthesis energy loss after aortic valve replacement (AVR) and compared with that of pressure gradient.

Methods 76 patients ( $72 \pm 7$ years, 46 males) underwent AVR for aortic stenosis were prospectively studied using echocardiography up to 3 years. Its mean systolic pressure gradient, effective orifice area and LV mass were determined by two dimensional Doppler echocardiography. Aortic sino-tubular junction diameter, aortic $\mathrm{EOA}$ and pressure gradient were used to calculate aortic valve energy loss (Garcia D, et al., Circulation 2000;101:765-71).

Results In aortic stenosis, its pressure gradient recovery was only $28 \pm 9 \%$. Thus, for mean pressure gradient of $41.0 \pm 16.7 \mathrm{~mm} \mathrm{Hg}$, the mean net energy loss is $32.1 \pm 14.3 \mathrm{~mm} \mathrm{Hg}$. Three years after $\mathrm{AVR}$, the mean pressure gradient fell to $7.7 \pm 3.5 \mathrm{~mm} \mathrm{Hg}$, and the energy loss reduced to $4.6 \pm 2.4 \mathrm{~mm} \mathrm{Hg}$, both $\mathrm{p}<0.001$ vs pre-AVR.
The percentage of pressure gradient recovery has increased to 61 $\pm 14 \%(p<0.001)$, which is inversely correlated with aortic sinotubular junction diameter and positively correlated with EOA $(p<0.01, p<0.01, r=0.90)$. LV mass index regressed from pre-AVR $\left(182 \pm 48 \mathrm{~g} / \mathrm{m}^{2}\right)$ towards normal level $\left(126 \pm 31 \mathrm{~g} / \mathrm{m}^{2}\right), \mathrm{p}<0.001$.

Conclusions AVR not only significantly reduced aortic valve pressure gradient itself, but has doubled the proportion of pressure recovery. This resulted in continuously low prosthesis energy loss and satisfactory LVH regression. As the sino-tubular junction diameter significantly affects aortic valve energy loss, restoring normal root geometry should be considered as one of the objectives for aortic valve surgery. 